\title{
Physiotherapists' Perceptions of Disability Awareness in KwaZulu-Natal
}

\author{
Joan Pather ${ }^{1}$, Stacy Maddocks ${ }^{1}$, Nomzamo Chemane $^{1} \&$ Verusia Chetty ${ }^{1}$ \\ ${ }^{1}$ Discipline of Physiotherapy, School of Health Sciences, Westville Campus, University of KwaZulu-Natal, \\ Durban, South Africa \\ Correspondence: Verusia Chetty, Discipline of Physiotherapy, School of Health Sciences, Westville Campus, \\ University of KwaZulu-Natal, Private Bag X54001, Durban 4000, South Africa. Tel: 27-31-748-905-279. E-mail: \\ chettyve@ukzn.ac.za
}

Received: June 24, 2018 Accepted: August 2, 2018 Online Published: November 12, 2018

doi:10.5539/gjhs.v10n12p83 URL: https://doi.org/10.5539/gjhs.v10n12p83

\begin{abstract}
Background: Disability awareness is essential for rehabilitation professionals, such as physiotherapists, to provide an understanding of disability and to influence environmental and attitudinal societal barriers. There is a paucity of literature on the perceptions of physiotherapists about disability awareness in the South African context.

Objectives: To explore the perceptions of physiotherapists on disability awareness in order to influence the rehabilitative care offered to people with disability.

Method: This study adopted an explorative, descriptive qualitative design. Two focus group discussions were conducted with public healthcare physiotherapists employed at urban and rural facilities. Transcribed data were analysed using conventional content thematic analysis. There were five participants in each group: two males and eight females, with nine participants having worked for more than six years.
\end{abstract}

Results: The discussions revealed five major themes: consequences of a dearth of knowledge; personal influence; facilitators of disability awareness; barriers to disability awareness; and recommendations.

Conclusion: Access to healthcare is hindered by a lack of understanding, and the subsequent behaviour, including the stigmatisation of people with disabilities. The study highlighted that poor knowledge of disability and lack of cultural sensitivity among physiotherapists working among communities strongly motivated by cultural beliefs, may influence the rehabilitation of people living with disability. It was concluded that self-reflection, coupled with deeper community engagement and continuing professional development, would enhance physiotherapy practice and disability awareness in the future.

Keywords: disability, disability awareness, physiotherapists, public healthcare

\section{Introduction}

Disability awareness by rehabilitation healthcare professionals is critical for the promotion of health and wellbeing and for offering good treatment to people with disabilities (PWD) (Frantz, 2008; Rao, Sharmila and Rishita, 2002). The concept is focused on providing knowledge and understanding of disabilities to communities serviced by health professionals (Culp, 2016). Disability awareness provides necessary information about PWD in order to curb misunderstandings, which often create barriers to social and environmental inclusion (Gupta \& Singhal, 2004; Masasa, Irwin-Carruthers, \& Faure, 2005). Furthermore, disability awareness aims to address the health promotion needs of PWD in order to prevent the onset of secondary and tertiary disease (Njoki, Frantz, \& Mpofu, 2007). The World Health Organisation (WHO) describes health promotion as a process of enabling people to increase control over, and improve, their health (WHO, 1986). For the purposes of this paper, we have used the international classification of functioning, disability and health (ICF) (WHO, 2001) in order to understand disability and disability awareness. The understanding of disability through the ICF framework stems from a biopsychosocial standpoint which factors in activity limitations and participation restrictions. Holistically, it takes into account the physiological, environmental and psychosocial aspects of the individual when managing the challenges faced by PWD (Masasa et al. 2005; Mlenzana et al. 2013). Disability awareness should be offered within health promotion strategies to reduce or eliminate attitudinal and environmental barriers which constrain, rather than support, the participation of PWD (Rao et al., 2002). 
In South Africa, physiotherapists study a four-year undergraduate degree before completing a mandatory community service year of practice. They are governed by the statutory requirements provided by the Health Professions Council of South Africa (HPCSA) (Professional Board-HPCSA, 2018). Physiotherapists, as rehabilitation professionals, are responsible for creating disability awareness, optimising physical function, and integrating PWD into communities (Frantz, 2008; Fricke, 2005). Health promotion is crucial to all physiotherapy-led patient interactions and treatment sessions (Walkenden \& Walker, 2015). The adequate provision of health promotion information to PWD in communities has been identified as a challenge in the effective implementation of primary health care (PHC) in South Africa (Ned, 2017). Physiotherapists are required to demonstrate knowledge of the likely consequences of disability and in-depth understanding of appropriate management strategies for disabilities in order to be professionally and socially responsive to PWD (Joseph, 2011).

Building a rapport with patients is crucial to promoting health behaviour change and should include aspects of religious and cultural sensitivity (Rollnic \& Mason, 1999). Meaningful engagement with PWD requires that they be active participants with health professionals in the conceptualisation and implementation of disability awareness in the community. The involvement of PWD allows for health professionals to draw from the lived experience of PWD in order to enhance their own understanding of the consequences of disability and to strengthen disability awareness (Wegner \& Rhoda, 2015). Physiotherapists are in a prime position to facilitate this collaboration, due to the ongoing interaction they have with PWD during the, often lengthy, courses of rehabilitation (Walkenden \& Walker, 2015).

The study aimed to explore perceptions of physiotherapists on disability awareness within one of the public healthcare settings in South Africa in order to influence the rehabilitative care offered to PWD.

\section{Methodology}

\subsection{Design}

The research adopted an explorative, descriptive qualitative design (Creswell et al. 2007) to understand in depth the perspectives of physiotherapists of disability awareness. The primary method of enquiry used in this study was focus group discussions (FGD's) (Hennink, 2013). FGD's channel interactional discussions to elucidate the details of complex experiences and rationalisations behind individuals' actions, beliefs and attitudes (Powell \& Single, 1996).

\subsection{Setting}

The study was conducted in the province of KwaZulu-Natal (KZN). The population of KZN is diverse in culture, language, race and ethnicity, comprising three dominant official languages, four race groups and a variety of cultures amongst the different race groups (Grut et al., 2012). KZN is the only province in South Africa for which provision for a monarchy is made in the constitution (Certification of the Constitution of the province of KwaZulu-Natal, 1996). Most communities in KZN are still disadvantaged in their access to resources and still maintain strong ethnic and cultural views about health and rehabilitation (Wegner \& Rhoda, 2015).

KZN has an 8.4\% prevalence of disability in a total population of 7349154 (Census 2011: Profile of persons with disabilities in South Africa, Report 03-01-59). At present, PWD access physiotherapy services in public institutions by referrals from other healthcare workers. PWD are rarely seen as self-referrals in public institutions. Presently, physiotherapy services are available in most hospitals and Community Health Centres in KZN.

\subsection{Participants}

The study population included physiotherapists employed by the Department of Health in public sector facilities across both urban and rural settings in KZN. Only qualified physiotherapists, currently registered with the Health Professionals Council of South Africa (HPCSA) for the years 2017-2018, and practising in KZN province, were included in the study. Purposive maximum variation sampling, including ethnicity, gender, geographic location and years of working experience, was used to identify participants from rural and urban healthcare facilities in KwaZulu-Natal. Eligible candidates were invited to participate in the study telephonically or via email. Although ten participants were initially recruited for each focus group discussion, the final sample included two groups (urban and rural), each of five participants. The participants were from the EThekwini (urban), Ugu (rural) and King Chetshwayo (rural) districts of KZN (See Table 1 below). The remaining candidates were unwilling to participate due to personal commitments on the day of the FGD's. Due to logistical and financial constraints, further FGD's could not be held to accommodate the remaining recruited participants. One FGD consisted of physiotherapists working in the rural areas and the other represented physiotherapists working in the urban areas. Pseudonyms are used when referring to physiotherapists in the discussion of the findings. 
Two of the ten participants were male therapists. Only one participant had less than five years of work experience in South Africa. Table 1 reflects the demographic characteristics of the physiotherapists.

Table 1. Demographics of Participants $(n=10)$

\begin{tabular}{llllll}
\hline $\begin{array}{l}\text { Participant } \\
\text { Pseudonym }\end{array}$ & Age range & Gender & $\begin{array}{l}\text { Years } \\
\text { Experience }\end{array}$ & $\begin{array}{c}\text { of } \\
\text { Context }\end{array}$ & Type of Facility \\
\hline Vani & $50-55$ years & F & 31 years & Urban & Regional Hospital \\
Sheila & $45-50$ years & F & 30 years & Urban & District Hospital \\
Dumi & $36-40$ years & F & 17 years & Rural & District Hospital \\
Enhle & $36-40$ years & F & 15 years & Rural & District Hospital \\
Bianca & $31-35$ years & F & 14 years & Urban & District Hospital \\
John & $31-35$ years & M & 10 years & Rural & Regional Hospital \\
Candice & $31-35$ years & F & 10 years & Urban & Specialised Rehabilitation \\
Santhuri & $31-35$ years & F & 8 years & Urban & District Hospital \\
Amy & $25-30$ years & F & 7 years & Rural & District Hospital \\
Thobani & $25-30$ years & M & 5 years & Rural & District Hospital \\
\hline
\end{tabular}

\subsection{Data Collection Procedure}

Two separate focus group discussions for physiotherapists working in urban and physiotherapists employed in rural facilities were conducted. A flexible focus group guideline was developed from the literature in accordance with the aims of the study (Powell \& Single, 1996) and piloted among physiotherapists in academia (Carey, 1995). Key words for the responses were noted and supporting literature was identified for each response. Thereafter, recommendations and modifications were applied to the guide prior to its use. The FGD's were conducted in English. A dictaphone and notebook were used to record participants' verbal and non-verbal responses. In attendance at each discussion was a facilitator to conduct the focus group discussion, a research assistant to record all non-verbal cues, and a moderator seasoned in qualitative research methods to oversee the quality of the procedure. The focus group discussions continued until no new information emerged and data saturation was reached. Checking was conducted with participants during the discussions by asking participants if the researcher's interpretation of their comments was accurate in moments of ambiguity or confusion. The results were sent to the participants' after the discussions, and after analysis, to ensure that the participant responses were accurately interpreted, in order to enhance trustworthiness of the data (Guba, 1981). Each discussion lasted approximately an hour.

\subsection{Data Analysis}

The FGD's were transcribed verbatim. The transcriptions were read and re-read in order to familiarise the researcher with the data and gain a full understanding of the content. Data was coded to identify common concepts discussed by the participants. Conventional thematic analysis was then used to identify, analyse and discuss emergent themes from the data (Boyatzis, 1998). Codes were then grouped into categories, analysed into sub-themes and finally broader themes were identified. These themes were discussed and debated with the moderator and an expert in qualitative research until consensus was reached. This process of analysis further strengthened the study's trustworthiness and rigor (Creswell et al., 2007).

\section{Findings}

\subsection{Emergent Themes}

Five major themes emerged from the two focus group discussions: consequences of a dearth of knowledge; personal influences; facilitators of disability awareness; barriers to disability awareness; and recommendations. Themes, sub-themes and participant quotes are presented in Table 2 below. 
Table 2. Themes, sub-themes and illustrative quotes

Consequences of a dearth of knowledge

\begin{tabular}{ll}
\hline Sub-theme & Quotes \\
\hline $\begin{array}{l}\text { Physiotherapists' } \\
\text { understanding }\end{array}$ & $\begin{array}{l}\text { "Because of lot of it comes from what society believes and what society grew up being told, even us - } \\
\text { we didn't engage or interact with children with disability until we were professionals." Vani (urban } \\
\text { physiotherapist) }\end{array}$
\end{tabular}

"My understanding of disability can be mentally or physical. So a disabled person can be differentiated from a more abled person by assessing their functions or mental capabilities." Thobani (rural physiotherapist)

“........ I think that, often, we as people in rehab tend to focus (especially physiotherapists), we focus a lot on physical disabilities, but there is lots of perceptions of disabilities, and I think that the community influence on disability is something that we all need to understand. And that's just about it." Sheila (urban physiotherapist)

"But the only reason ICF was brought into us, is when the students started coming to our institutions and they ask about you ICF and we were like: "What is that we use the Barthel Index, we use

Community interpretation

Healthcare knowledge

Stigmatisation Frankels and ASIA scales and then you're finding older therapists battling to understand the participation and environment factors. If there is something new brought into the university it should be brought to the hospitals. So it wasn't and it still isn't. Now it is hard to bridge the gaps........" Sheila (urban physiotherapist)

"...... then you have to look at the legislation because the legislation pertains to so many other sectors...... So if we look at the legislation according to health, we do take that into account, is just that with everything else. Like with disability, it's a person, its health. So it is all the sort of different sectors you have to include. But then do we base our health promotion on general, or should we should we do it in terms of multi-disciplinary, where we have other sectors being involved, particularly in health promotion......? ” John (rural physiotherapist)

staff "....there are certain terms that are given to disabled people that are derogatory, that when you analyse the meaning, it is an insult. They come from the lack of understanding of disability and for example 'umuntu ono isishawa', a person with albinism, isishawa. In other words, a person has been hit or punished.” Dumi (rural physiotherapist)

" people believe that it's witchcraft or curse on people who have been bad" Enhle (rural physiotherapist)

"I happen to sit in the Complaints Committee of the Hospital and the complaints that are coming from the people with disability, the offenses committed by the so-called people that should be understanding what disability is, it amazes me." Bianca (urban physiotherapist)

"People are going to lose their jobs, simply because they don't understand what disability is. They discriminate against disability; people are going to lose their jobs.” John (rural physiotherapist)

\begin{tabular}{ll}
\hline Personal Influences & Quotes \\
\hline Sub-theme & "Instead of just focusing on the people with disabilities and the community, let us also focus on \\
ourselves working in the community. Because a lot of people, they might know what disability is, but & they might not understand it." Candice (urban physiotherapist) \\
& It goes back to your personality, what kind of a person are you? If you are a person that would go all \\
& the way just to help people, until that person gets better, then that is the drive behind the awareness \\
& campaigns." Enhle (rural physiotherapist) \\
"I think all the therapists are comfortable where they are... people don't want to move from their \\
\\
comfort zone ... to go to the community. It is trying to motivate them, make them to realize how \\
important it is to reach out to go out there even in an urban setting .... The rural therapists are very \\
proactive with that, but the urban ones, oh well...this is where we are." Candice (urban \\
physiotherapist) \\
"...once you see what people are going through, and the pain of a mother says that even her in-laws \\
are treating her this bad because they do not understand. That drives me to create awareness."
\end{tabular}


Santhuri (urban physiotherapist)

"Then you see that person walking into your department (following disability awareness Experience intervention). That is a positive reinforcement for me and it is a personal experience." Amy (rural physiotherapist)

"It is exposure. It is experience. With experience you will know how to deal with an emotional disabled person.” Bianca (urban physiotherapist)

"Yes, generally you would think that the older you get, the more knowledge and experience you get so that informs you better about the situation." Santhuri (urban physiotherapist)

Facilitators of disability awareness

\begin{tabular}{lrr}
\hline Sub-theme & \\
\hline Involvement & of \\
people & with $\quad c$ \\
disabilities & & "
\end{tabular}

Quotes

Disability forums

Barriers to disability awareness

\begin{tabular}{ll}
\hline Sub-theme & Quotes \\
\hline Facility & "So, we are not looking at disability further than the hospital, unless you are in a rural setting and \\
infrastructure & you are rostered to go out." Santhuri (urban physiotherapist) \\
& "I think the services we are asked to provide in institutions makes the impact on what we can do. Our \\
& priority in a hospital is in-patients and acute care. So now with the situations as it is, very limited \\
& posts are vacant and you can fill them. So you just have to prioritize those services." Enhle (rural \\
& physiotherapist)
\end{tabular}

"But going out and doing it, is something we hardly do and I am not giving excuses, but the issue of resources in some hospitals: when you out, you close the department." Bianca (urban physiotherapist)

Resource limitations

"The current situation does not allow us to take the services to where we want to take it." Sheila (urban physiotherapist)

"In the country, we haven't done enough research about disability, it's all based on the western knowledge. But the content of the western civilization or countries it is different from the African context." Thobani (rural physiotherapist)

"I think that gender would influence it, depending on who you are. If you go to certain rural areas, you cannot address traditional leadership if you are a female... And you are only allowed to speak when spoken to in some areas." Enhle (rural physiotherapist)

Environmental systems

\section{Lack of intersectoral engagement}

"So out here in our district we know that we don't have the interaction with the disability community...... Yes they exist, but it almost seems as if they want to function independently as much as we've had meetings with them also... But neither do they invite us." Dumi (rural physiotherapist)

"We are aware of the traditional healers, but we don't involve them in our programmes ... We do not include it (traditional healing) in our material, you know the knowledge of it. We just put everything that is pertaining to physio or any other rehab in the material that we use" Vani (urban physiotherapist)

"If you are talking about disability, attitudes of physiotherapists, I think that it is a bigger thing than that. I think it is the attitudes of the entire health team. .... A lot of us may even understand disability and what our role is in disability, but we are not able to exercise it, because the control system by the doctor - he is always ready to send anybody home - is not giving you the chance to even do 
anything. ” John (rural physiotherapist)

Lack of "But I think that the awareness of disability and how it impacts on a person's life and everything that multidisciplinary collaboration goes afterwards ... needs to be done with the medical profession in our settings....doctor feels that he treats the patient and he gives the medication and the patient must go and that is the end of story." Vani (urban physiotherapist)

\section{Recommendations}

Sub-theme

Task-shifting

Improved

stakeholder involvement

Materials and media

Innovative ideas

Education sharing

\section{Curriculum review}

\section{Quotes}

"I know that at one stage they (referring to Department of Health) used to have the CBR (referring to Community Based Rehabilitation) workers. It is something that we need to do again. Because it is specific to rehab." Thobani (rural physiotherapist)

"So we have to educate community caregivers (CCGs), if we can't physically go to the community. We can educate them on disability and programmes, then we can filter to the houses that cannot come, because of transport and costs. " Dumi (rural physiotherapist)

"Whilst we are running awareness and everything that there are key people that are usually left behind that I think should be in the forefront: we have the traditional leaders, the traditional healers, we have the religious leaders..." Enhle (rural physiotherapist)

"As much as we would like to advocate for disability issues, there needs to be buy-in by the disability community.” Amy (rural physiotherapist)

"Consultation is very important...engaging..... representation not only from the communities, but also from government sectors like the Department of Labour, the Departments of Transport, Social Development, Health, Education. There is lot of issues that need to be addressed." John (rural physiotherapist)

"The posters - students create posters and sometimes they do research on the topic." Dumi (rural physiotherapist)

"Through the media; whether it is television or radio...there's got to be some awareness that goes through media. People talk about what they see on TV and/or listen to on radio and take it as it is." Thobani (rural physiotherapist)

“...educational pamphlets for various topics such as disability awareness....in English and IsiZulu. ” Bianca (urban physiotherapist)

“...research articles, the internet..." Santhuri (urban physiotherapist)

"We're using some of the SASP materials. We make our own posters." Sheila (urban physiotherapist)

"I think that something like a sketch or a drama, because sometimes if you just go there and tell them the theory, you are just wasting your time. It's better to put in practical so that they can see." Enhle (rural physiotherapist)

"We can also communicate via churches. We can ask them that we are going to teach the Christians about the disabilities and maybe the schools can also come........ They still need to be taught about disability. ” John (rural physiotherapist)

"I think it is the sharing of knowledge among the public at large; in a form of education: teaching people about disability, what disability is and how to manage people with disability at home, school or the work place." Vani (urban physiotherapist)

"So it is important to teach the family, even the caregiver, that this is how you treat a disabled person." Sheila (urban physiotherapist)

"So when we are talking about the curriculum, be it for nurses, therapists or even doctors, it is the medical model, as the medical model is disease-based. So if I am born with Spinal Bifida they approach it medically ...should interact with social inclusion. ” Dumi (rural physiotherapist)

"I am thinking that the DCT (referring to local physiotherapy placement in rural settings), if anything, will provide much better exposure to our students, anyway. So when you are talking about education, their skills and training out, there will be a better exposure to disability." Bianca (urban physiotherapist) 


\section{Discussion}

The five major themes, consequences of a dearth of knowledge; personal influences; facilitators of disability awareness; barriers to disability awareness; and recommendations are discussed with a focus on sub-themes. The discussion is framed within the context of South African contemporary literature in healthcare, predominantly drawing from similarly resourced areas of study.

\subsection{Consequences of a Dearth of Knowledge}

The overarching theme, consequences of a dearth of knowledge, included the subthemes: physiotherapists' understanding, community interpretation, healthcare staff knowledge and stigmatisation. Some physiotherapists commented that their experiences and exposure during their formative years influenced their knowledge and their current practice. Many physiotherapists in this study indicated an understanding of disabilities from a biomedical standpoint. Most of the physiotherapists reported not using the international classification of functioning (ICF) in the rehabilitation of PWD, as they did not have adequate knowledge about it. The ICF was introduced in South Africa in 2006, as a way of understanding the complex interaction between the biological, psychological, cultural and social factors of disability (WHO, 2001). There appeared to be inadequate training in, and development of, the ICF for physiotherapists who qualified prior to 2006; hence the lack of knowledge in this area. Knowledge and application of the ICF is crucial to rehabilitation, particularly in a setting where the diversity of geography, infrastructure, culture and ethnicity demands that health professionals understand, in context, limiting factors and participation restrictions to the rehabilitation of disabilities (Masasa et al., 2005).

Physiotherapists in the study demonstrated a lack of knowledge of the legislation that governs the rights of PWD. With the reforms in South Africa in 1994, and the creation of the UN Millennium Development Goals in 2000, there have been adjustments to the existing health policies and the development of new ones. The National Rehabilitation Policy (DoH, 2000) encouraged community-based services and the primary healthcare (PHC) approach to service delivery. Extreme challenges were experienced in the implementation of this approach in South Africa as a result of healthcare workers still adopting the curative-oriented medical health service model (Mji et al., 2013). This could have been attributed to healthcare workers' lack of knowledge with regards to the policy changes; and to inadequate skills to implement the PHC principles according to the psychosocial and medical models at the time.

All the physiotherapists in this study agreed that the multidisciplinary healthcare team lacked knowledge they believed was critical to disability awareness issues. Badu et al. (2016) found that doctors and nurses in Bhutan had a less positive attitude towards PWD, and were not at ease with disability and rehabilitation issues because they had less knowledge of these matters. The lack of knowledge about disability and rehabilitation issues leads to a lack of compassion and sensitivity in understanding PWD, resulting in poor, or less positive, attitudes towards them in the provision of healthcare. The emergence and discussion of knowledge as a theme reflects the influence it could have on the rehabilitation framework, including awareness programmes offered to PWD by the professionals in multidisciplinary teams.

The study by Masasa et al. (2005) demonstrated that caregivers of PWD displayed varying amounts of knowledge of disabilities, depending on their level of exposure to PWD in previous years. Although the groups were heterogeneous, they were similarly influenced by previous exposure to, and knowledge about, disability. Physiotherapists indicated that the communities they served believed in certain myths, such as disability being a punishment or result of witchcraft. Several studies corroborate that cultural beliefs contribute to health seeking and participatory behaviour; and these beliefs can leave PWD and their families feeling marginalised and stigmatised within the communities they reside (Hussey et al., 2017; Masasa, 2005; Wegner \& Rhoda, 2015). Such behaviour indicates a lack of education in the community about disability: people do not understand the biopsychosocial causes of disabilities and the interventions available to manage PWD. IsiZulus in KZN maintain a great deal of respect for their elders and thus obey the cultural teachings from elders in the community. It is therefore essential that appropriate education and awareness about disabilities and PWD be provided to the communities. This necessitates that healthcare professionals, such as physiotherapists, possess cultural knowledge and competence (Wegner \& Rhoda, 2015). It is imperative that physiotherapists acquire an understanding of the culture and beliefs within a community in order to develop skills to collaborate with communities in developing the strategies to promote disability awareness. The success of developing disability awareness is dependent on its relevance to cultural beliefs embedded in communities (MacLachlan, 2006). Robey et al. (2013) found that health science students demonstrated that cultural competency is best acquired by students' exposure to PWD within their cultural contexts, which will consequently acquaint them with the daily needs and realities of PWD. Furthermore, rehabilitation and programme outcomes are influenced by the cultural beliefs held by PWD, families and 
communities. If these are not incorporated into rehabilitation approaches, it could lead to a lack of engagement (Wegner \& Rhoda, 2015; Grut et al., 2012). Physiotherapists believed that stigma around disability still seems to plague awareness, and was thought to be career threatening, especially when such practices are demonstrated by the team responsible for offering comprehensive rehabilitative care to PWD. The participants in this study further indicated that exposure to community-based rehabilitation benefits both the community and the physiotherapist. The community becomes more aware of disability, whilst the physiotherapist develops professionally. They further stated that greater support is needed to overcome the barriers that limit their services institutionally. Mostert-Wentzel, Frantz and Van Rooijen (2013), in support of this finding, indicated that the absence of in-service learning through conducting community work in diverse contexts such as homes, clinics, schools and hospitals, as a result of system and structural barriers, impedes professional development.

\subsection{Personal Influences}

The second major theme, personal influences, encompassed introspection, self-motivation, compassion, fulfilment and experience of physiotherapists. The physiotherapists believed that introspection is important to really understand disability issues. They also felt that motivation was key to giving the best as a practitioner. Personal fulfilment in providing holistic care was also a contributor to including awareness in practice, as well as experience as a clinician. The possession of these attributes allows for effective and efficient delivery of healthcare and rehabilitation services throughout communities in the vast rural areas of KZN, despite the scarcity of resources. In a study by Cobbing et al. (2017), the perceptions of community healthcare workers were explored following their implementation of a home-based rehabilitation programme for people with HIV living with disabilities. Participants reported feeling a sense of self-worth and empowerment from offering care to PWD. Professional attributes of healthcare professionals, such as creativity, perseverance, patience and diplomacy when working with PWD in the community, contributed towards the development and success of treatment, with reported levels of increased job satisfaction (Twible \& Henley, 2000; Jain et al., 2009). Similarly, physiotherapists in our discussions felt fulfilment when making a difference in communities through awareness programmes.

\subsection{Facilitators of Disability Awareness}

The theme, facilitators of disability awareness, includes the sub-themes, involvement of people with disabilities and disability forums. The therapists agreed that developing disability awareness, in their experience, necessitates the involvement of PWD. According to Mji et al. (2013), the legislation guiding the discourse on South African rehabilitation requires the full involvement of PWD at all levels of care offered. Some therapists commented that their service communities have disability forums with representatives from the community, that allow for advocacy in strategies to promote disability awareness. Several studies have highlighted the principles of CBR that involves PWD, their families and communities when offering rehabilitation or health initiative programmes in a health promotion approach (Mji et al., 2013). Often, the voices of these crucial role players are omitted and health awareness initiatives are not adopted or implemented (Kautzky \& Tollman, 2008; Naledi et al., 2011; Mji et al., 2013). This could be contributing to one of the reasons why health promotion is disregarded as a focus area in rehabilitation. Flint (2015) and Moshabela et al. (2016) concluded that the dominant institution-based healthcare system resulted in the patients becoming passive beneficiaries of healthcare services, thus encouraging ongoing dependency in the expert-driven and standardised approach to healthcare. Inputs from the community were not encouraged and no attention was given to how communities managed healthcare in the absence of a healthcare practitioner. Ned et al. (2017) commented on the lack of patient-centred, contextually targeted, health promotion and disease prevention strategies offered for PWD. The outcome of this deficit is PWD who are not actively participating in, or making contributions to, their healthcare planning.

Another reason for the poor uptake of health awareness initiatives could be linked to our rehabilitation practices which focus on the biomedical model, with emphasis on the individual, rather than on the biopsychosocial model that includes community and societal factors (Hammel et al., 2015; Kumurenzi, 2011; Terzi, 2004).

\subsection{Barriers to Awareness of Disability}

The overarching theme, barriers to awareness of disability, incorporated the sub-themes of facility infrastructure, resource limitations, environmental systems, lack of inter-sectoral engagement and lack of multidisciplinary collaboration. Some of the therapists believed that the hospital structure and 'how things were done' historically dictated the levels of involvement in community outreach strategies like disability awareness. This impedes the physiotherapist's ability to advocate for, and motivate, inter-sectoral collaboration that could improve patient referrals to the relevant people, encourage sharing of resources, and initiate community-based and community-led disability awareness. Resource limitations, such as staff shortages, were also believed to influence the implementation of disability awareness. Several studies (Chetty, 2015; Ned et al., 2017) have commented that staff 
shortages and resource limitations hinder the provision of optimal care to PWD. The health system in South Africa is inequitable in that the majority of the population access public healthcare from overburdened facilities, and this puts extra strain on the healthcare professionals servicing these care facilities (Chetty, 2015; Cobbing et al., 2013; Naledi et al., 2011; Ned et al., 2017).

One participant believed that issues of disability awareness were still based on western knowledge, which has less relevance in Africa, and South Africa in particular. Owusu-Ansah and Mji (2013) stimulated awareness around African indigenous knowledge, based on research. They aimed to overcome cultural barriers through a shift from western methodologies to the adoption of a more 'Afrocentric' approach to developing research centred on PWD. The inclusion of culturally sensitive approaches in, for example, the disability awareness of rehabilitation professionals should facilitate improved participation and uptake by PWD and their communities. Ned et al. (2017) agree that indigenous health knowledge lies inactive within the communities we serve. Some therapists felt that a lack of inter-sectoral collaboration between the community, the disability sector and healthcare professionals inhibits optimal healthcare and rehabilitation. Ned et al. (2017) also believed that gaps in inter-sectoral collaboration contributed to the flaws in South African healthcare systems. Hussey et al. (2017), after research in South Africa, revealed that PWD and their representatives felt that the government sectors do not function cohesively and this has contributed to political barriers to the optimal implementation of the United Nations Convention on the Rights of Persons with Disabilities (UNCRPD) in the country. Furthermore, participants in Hussey's study felt that there was a lack of consultation with PWD and community leaders by politicians. A physiotherapist in our study believed that traditional healers were omitted from efforts to improve disability awareness and develop appropriate programmes. Traditional healers and religious leaders are vital avenues to create pathways for communication, discussion and dissemination of information, as they are highly esteemed and respected members of the community (Masasa et al., 2005). Research indicates a lack of recognition of the medical pluralism of healthcare in South Africa (Flint, 2015; Moshabela et al., 2016). Ned et al. (2017) present narratives on the obligatory legislative commitments made to integrate traditional healers into South African healthcare systems since 2013, yet a gap is still evident in translating the policies into practice.

On a meso-system level, the lack of multidisciplinary collaboration has damaged healthcare systems; in turn, affecting disability awareness issues. The teamwork approach is essential to optimise care. A lack of knowledge of disability awareness among team members will inadvertently affect service delivery to PWD. Input is needed from all team players to ensure that they maintain the same approach towards PWD. If team members do not collaborate it impacts on rehabilitation outcomes (Chetty \& Maharaj, 2013). Chetty and Maharaj, (2013), in their work on collaboration between healthcare professionals, suggested that partnerships between the multidisciplinary teams, as well as knowledge of the role of team members, is crucial in the care offered. In KZN, PWD have challenges with accessing rehabilitation and other healthcare services, due to transport and financial constraints. It is essential that team members are able to communicate with one another in ensuring that the patient gets value for money. Maddocks et al. (2017), in an exploration into collaboration between doctors and physiotherapists in the rehabilitation of people living with HIV and with disabilities, believed that a cohesive partnership between healthcare professionals would influence the care offered. Participants in our study reported that they were often not included in the clinical decision-making process for clients who were in-patients at higher care-level facilities, especially regarding readiness for discharge. Collaborative decision-making for PWD involving multidisciplinary and multi-sectoral engagement amongst all role players is critical for improved outcomes of these patients (Bruner et al., 2011).

\subsection{Recommendations}

Finally, the recommendations offered by physiotherapists included task-shifting; improved stakeholder involvement; using materials and the media; innovative ideas; education sharing; and curriculum review. Some physiotherapists believed that implementing rehabilitation in the community, or using mid-level workers, could ameliorate staffing issues and offer respite to overburdened facilities. By using these workers in every Ward-Based Outreach Team (WBOT), they could support generalist healthcare workers in identifying disability issues and referring appropriately, utilising the CBR approach (Chetty, 2015; Sherry, 2015). Lay personnel would offer rehabilitation and care to PWD under the supervision of qualified health professionals (Luruli et al., 2016). This is often termed 'task shifting' and has been advocated in similar contexts (Chetty, 2015; Cobbing et al., 2017; Ned et al., 2017). Empowering PWD through this approach would offer further respite as a strategy that would provide a platform for advocacy and integration of issues and societal barriers faced in communities (Ned et al., 2017; Grut et al., 2012). One therapist in our study believed that the task-shifting approach will offer continuity of care into the home (Cobbing et al., 2017). A study by Cobbing et al. (2017), in a similar context, found that using a task-shifting, home-based rehabilitation protocol proved to be beneficial to PWD, as well as the trained community healthcare 
personnel. The authors of this study believe that this involvement will improve the disability awareness strategies as the contextual factors and community knowledge will also become an imperative.

All the therapists agreed that collaboration between stakeholders, and inter-sectoral involvement, was critical in addressing disability issues. Collaborative approaches to rehabilitation have the potential to offer seamless care to PWD (Bruner et al., 2011; Chetty \& Maharaj, 2013; Maddocks et al., 2017; Ned et al., 2017). Greater effort needs to be made to overcome professional hierarchies and to encourage an inter-professional, collaborative approach that considers the participation of all team members in clinical decision-making (Chetty \& Maharaj, 2013; Maddocks et al., 2017). Multidisciplinary teamwork focuses on the clients' needs, while mutual respect, power, goals and partnerships are shared among team members (Bruner et al., 2011; Gerdes et al., 2001). In this study, the team could be actively involved in implementing the awareness programmes, as well as the development of the most effective strategies in collaboration with PWD and community leaders.

A few therapists believed that materials and the media could offer creative interventions to disability programmes. An effective disability awareness programme should have clear objectives and should focus on a particular audience, choosing the media that matches the needs of the multicultural and multilingual society (Hugo \& Smit, 1999). The Health Education, Appropriate Media and Technology, and Socio-Cultural sensitivity (HAMSOC) model offers some direction to assist with the planning and drawing up of messages to promote disability awareness within a multicultural setting, and should be applied by physiotherapists (Hugo \& Smit, 1999). The HAMSOC model looks at the medium used for the delivery of information, which is communicated whilst being sensitive to the socio-cultural implications in the content of the information. Limited resources and the socio-cultural issues in KZN makes the use of the HAMSOC model an ideal one to adopt in promoting disability awareness. Additional sources, such as social development, housing, transport, education, advocacy and support groups from government sectors, non-governmental organisations and peers are also believed to be good sources of information (Twible \& Henley, 2000). These sources of information should be augmented by collaborative information sharing with PWD and key community players (Grut et al., 2012) who consider the contextual and social issues of the community, rather than just the challenges of PWD in order to promote holistic health and wellness (Ned et al., 2017). Another therapist believed that innovative platforms should be created in order to capture the attention of the communities. Education of communities on various fora was also felt to be an avenue to communicate knowledge. Schools, religious facilities and gatherings were suggested as platforms for education. Furthermore, the undergraduate programmes offered to healthcare professionals were suggested by participating therapists as an ideal setting to influence the future multidisciplinary team's approach to disability issues and awareness. Many studies in South Africa that have focused on the care offered to PWD recommend the need to review undergraduate programmes and in-service training approaches in order to facilitate appropriate clinical training that addresses the needs of the communities (Mji et al., 2013; Ned et al., 2017; Twible \& Henley, 2000).

\section{Conclusion}

This study has provided insight into the perceptions of physiotherapists on disability awareness. Participants believed that poor knowledge about disabilities and disability awareness among healthcare workers and community members contributes significantly to the gap that exists in the implementation of disability awareness. Physiotherapists and other team members who lacked knowledge about disability issues displayed poor attitudes towards PWD, which was reflected in their actions. Updated training and development is required to upskill physiotherapists so that they keep abreast of changes and are able to effectively promote disability awareness. Physiotherapists must be introspective and understand other religions and cultures to take care that they do not impose their religious and cultural beliefs on the community. They need to work in collaboration with the community. Facilitators identified in the study must be strengthened by more active involvement of physiotherapists in community-based rehabilitation. The personal factors indicated by the physiotherapists in the study can be adopted by individuals to assist in overcoming some of the barriers identified in the study. Physiotherapists believed that task-shifting and improved stakeholder involvement is crucial for the successful promotion of disability awareness. Improved disability awareness can alter the perceptions of families and communities toward PWD, and has the potential to reduce barriers in society (Gupta and Singhal, 2004). Physiotherapists, playing an integral role in the rehabilitation of PWD, can influence such change by the manner in which disability awareness is approached and implemented.

\section{Competing Interests Statement}

The authors declare that there are no competing or potential conflicts of interest. 


\section{References}

Badu, E., Opoku, M. P., \& Appiah, S. C. (2016). Attitudes of health service providers: The perspective of people with disabilities in the Kumasi Metropolis of Ghana. African journal of disability, 5(1). https://doi.org/10.4102/ajod.v5i1.181

Boyatzis, R. E. (1998). Transforming qualitative information: Thematic analysis and code development. Sage.

Bruner, P., Davey, M. P., \& Waite, R. (2011). Culturally sensitive collaborative care models: Exploration of a community-based health center. Families, Systems, \& Health, 29(3), 155. https://doi.org/10.1037/a0025025

Chetty, V. (2014). A model of care for the rehabilitation of people living with HIV in a semi-rural South African setting. South African Heal Rev, 4(14), 183. https://doi.org/10.1007/s11606-014-2834-9

Chetty, V., \& Maharaj, S. S. (2013). Collaboration between health professionals in the era of antiretroviral therapy. Journal of the Association of Nurses in AIDS Care, 24(2), 166-175. https://doi.org/10.1016/j.jana.2012.04.005

Creswell, J. W., Hanson, W. E., Clark Plano, V. L., \& Morales, A. (2007). Qualitative research designs: Selection

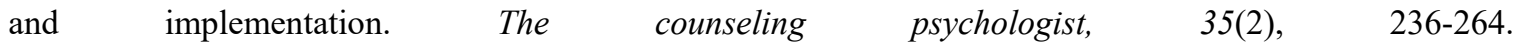
https://doi.org/10.1177/0011000006287390

Cobbing, S., Chetty, V., Hanass-Hancock, J., \& Myezwa, H. (2017)."Knowing I can be helpful makes me feel good inside, it makes me feel essential": community health care workers' experiences of conducting a home-based rehabilitation intervention for people living with HIV in KwaZulu-Natal, South Africa. AIDS care, 29(10), 1260-1264. https://doi.org/10.4102/phcfm.v9i1.137

Cobbing, S., Chetty, V., Hanass-Hancock, J., Jelsma, J., Myezwa, H., \& Nixon, S. A. (2013). The essential role of physiotherapists in providing rehabilitation services to people living with HIV in South Africa. South African Journal of Physiotherapy, 69(1), 22-25. https://doi.org/10.4102/sajp.v69i1.368

Culp, A. G. (2016). Disability Awareness: Knowledge, Attitudes, Behaviors, Self-efficacy, and Intentions among College Students (Doctoral dissertation, University of Cincinnati).

Flint, A. (2015). Traditional healing, biomedicine and the treatment of HIV/AIDS: contrasting south african and native American experiences. International journal of environmental research and public health, 12(4), 4321-4339. https://doi.org/10.3390/ijerph120404321

Frantz, J. M. (2008). Advocating evidence based health promotion in physiotherapy. South African Journal of Physiotherapy, 64(1), 28-30. https://doi.org/10.4102/sajp.v64i1.97

Fricke, M. (2005). Physiotherapy and primary health care: evolving opportunities. Winnipeg: Manitoba Branch of the Canadian Physiotherapy Association, college of Physiotherapists of Manitoba, department of Physical therapy, School of Medical Rehabilitation, university of Manitoba, 62.

Gerdes, J. L., Yuen, E. J., Wood, G. C., \& Frey, C. M. (2001). Assessing collaboration with mental health providers: The primary care perspectives. Families, Systems, \& Health, 19(4), 429. https://doi.org/10.1037/h0089470

Grut, L., Mji, G., Braathen, S. H., \& Ingstad, B. (2012). Accessing community health services: challenges faced by poor people with disabilities in a rural community in South Africa. African Journal of Disability, 1(1). https://doi.org/10.4102/ajod.v1i1.19

Guba, E. G. (1981). Criteria for assessing the trustworthiness of naturalistic inquiries. Ectj, 29(2), 75.

Gupta, A and Singhal, N. (2004). Positive perception in parents of children with disabilities. Asia Pacific Disability Rehabilitation Journal, 15(1), .22-35.

Hammel, J., Magasi, S., Heinemann, A., Gray, D. B., Stark, S., Kisala, P., ... \& Hahn, E. A. (2015). Environmental barriers and supports to everyday participation: a qualitative insider perspective from people with disabilities. Archives of physical medicine and rehabilitation, 96(4), 578-588. https://doi.org/10.1016/j.apmr.2014.12.00

Hatcher, A. M., Onah, M., Kornik, S., Peacocke, J., \& Reid, S. (2014). Placement, support, and retention of health professionals: National, cross-sectional findings from medical and dental community service officers in South Africa. Human resources for health, 12(1), 14. https://doi.org/10.1186/1478-4491-12-14

Hennink, M. M. (2013). Focus group discussions. Oxford University Press.

Health Professional Council of South Africa (HPCSA) (http://www.hpcsa.co.za/PBPhysiotherapy/Rules, 7 Apr 2018 17:34:14 GMT) 
Hugo, J., \& Smit, M. J. (1999). Rethinking the appropriateness of health education messages: Problems, principles and guidelines.

Hussey, M., MacLachlan, M., \& Mji, G. (2017). Barriers to the implementation of the health and rehabilitation articles of the United Nations convention on the rights of persons with disabilities in South Africa. International journal of health policy and management, 6(4), 207. https://doi.org/10.15171/ijhpm.2016.117

Jain, M., Mathur, A., Joshi, S., Goklani, P., Kothari, B., Prabu, D., \& Kulkarni, S. (2009). Job satisfaction assessment among dentists and dental auxiliaries in India. Journal of Dental Science, 7(2).

Joseph, P. M. (2011). Knowledge, attitudes and practices of physiotherapists regarding their role in health promotion in Gauteng Province, South Africa (Doctoral dissertation, University of Limpopo (Medunsa Campus).

Kautzky, K., \& Tollman, S. M. (2008). A perspective on Primary Health Care in South Africa: Primary Health Care: in context. South African health review, 2008(1), 17-30.

Kumurenzi, A. (2011). Rehabilitation services of persons with disabilities: Experiences of patients and service providers in a rehabilitation Centre in the Western Cape Province.

Luruli, R. E., Netshandama, V. O., \& Francis, J. (2016). An improved model for provision of rural community-based health rehabilitation services in Vhembe District, Limpopo Province of South Africa. African journal of primary health care \& family medicine, 8(2), 1-10. https://doi.org/10.4102/phcfm.v8i2.980

MacLachlan, M. (2006). Culture and health: A critical perspective towards global health. John Wiley \& Sons.

Maddocks, S., Chetty, V., Maghoo, A., Mhlongo, N., Mthembu, N., Khanyile, S., ... \& Mazibuko, S. (2018). 'Treating a patient should be approached in a holistic manner': collaboration of doctors and physiotherapists in the rehabilitation of people living with HIV. South African Family Practice, 60(2), 53-57. https://doi.org/10.1080/20786190.2017.1382969

Masasa, T., Irwin-Carruthers, S., \& Faure, M. (2005). Knowledge of, beliefs about and attitudes to disability: implications for health professionals. South African Family Practice, 47(7), 40-44. https://doi.org/10.1080/20786204.2005.10873260

Mji, G., Chappell, P., Statham, S. B., Mlenzana, N., Goliath, C., De Wet, C., \& Rhoda, A. (2013). Understanding the current discourse of rehabilitation: With reference to disability models and rehabilitation policies for evaluation research in the South African Setting. https://doi.org/10.4102/sajp.v69i2.22

Mlenzana, N. B., Frantz, J. M., Rhoda, A. J., \& Eide, A. H. (2013). Barriers to and facilitators of rehabilitation services for people with physical disabilities: A systematic review. African journal of disability, 2(1). dhttps://doi.org/10.4102/ajod.v2i1.22

Moshabela, M., Zuma, T., \& Gaede, B. (2016). Bridging the gap between biomedical and traditional health practitioners in South Africa. South African health review, 2016(1), 83-92. https://doi.org/10.1136/sextrans-2016-052974

Mostert-Wentzel, K., Frantz, J., \& Van Rooijen, A. J. (2013). A model for community physiotherapy from the perspective of newly graduated physiotherapists as a guide to curriculum revision. African Journal of Health Professions Education, 5(1), 19-25. https://doi.org/10.7196/ajhpe.203

Ned, L., Cloete, L., \& Mji, G. (2017). The experiences and challenges faced by rehabilitation community service therapists within the South African Primary Healthcare health system. African Journal of Disability (Online), 6, 1-11. https://doi.org/10.4102/ajod.v6i0.311

Naledi, T., Barron, P. \& Schneider, H. (2011). Primary health care in SA since 1994 and implications of the new vision for PHC Re-engineering. In A. Padarath \& R. English (eds.), South African health review, Health Systems Trust, Durban. Retrieved 03 February 2016, from http://www.hst.org.za/publications/south-african-healthreview- 2011

Njoki, E., Frantz, J., \& Mpofu, R. (2007). Health-promotion needs of youth with a spinal cord injury in South Africa. Disability and Rehabilitation, 29(6), 465-472. https://doi.org/10.1080/09638280600841224

Owusu-Ansah, F. E., \& Mji, G. (2013). African indigenous knowledge and research. African Journal Rao, A. J., Sharmila, M., \& Rishita, N. (2002). Awareness of and attitudes to disability in rural and urban communities of Andhra Pradesh, India: A comparative study. International Journal of Disability, Community and Rehabilitation, 2, 1-2. 
Owusu-Ansah, F. E., \& Mji, G. (2013). African indigenous knowledge and research. African Journal of Disability, 2(1). https://doi.org/10.4102/ajod.v2i1.30

Powell, R. A., \& Single, H. M. (1996). Focus groups. International journal for quality in health care, 8(5), 499-504. https://doi.org/10.1093/intqhe/8.5.499

Robey, K. L., Minihan, P. M., Long-Bellil, L. M., Hahn, J. E., Reiss, J. G., Eddey, G. E., \& Alliance for Disability in Health Care Education. (2013). Teaching health care students about disability within a cultural competency context. Disability and Health Journal, 6(4), 271-279. https://doi.org/10.1016/j.dhjo.2013.05.002

Sherryi, K. (2014). Essential considerations for equitable, accessible and poverty-reducing health care in South Africa. South African Health Review 2014/15, 89.

Terzi, L. (2004). The social model of disability: A philosophical critique. Journal of applied philosophy, 21(2), 141-157. https://doi.org/10.1111/j.0264-3758.2004.00269

Wegner, L., \& Rhoda, A. (2015). The influence of cultural beliefs on the utilisation of rehabilitation services in a rural South African context: Therapists' perspective. African journal of disability, 4(1). https://doi.org/10.4102/ajod.v4i1.128

World Health Organization [WHO]. (1986). Ottawa charter for health promotion. Health promotion, 1, iii-v.

World Health Organization [WHO]. (2010). Towards a common language for functioning, disability and health. 2002. Author: Geneva, Switzerland.

\section{Copyrights}

Copyright for this article is retained by the author(s), with first publication rights granted to the journal.

This is an open-access article distributed under the terms and conditions of the Creative Commons Attribution license (http://creativecommons.org/licenses/by/4.0/). 